TISSUE $\mathrm{pH} / \mathrm{pCO2}$ AND CONTINUOUS BASE EXCESS MONITORING

IN THE HUMAN FETUS

\title{
T. Weber
}

Department of Obstetrics and Gynecology, Hvidovre Hospital, University of Copenhagen, Denmark

C. Nickelsen

Department og Obstetrics and Gynecology, Rigshospitalet, University of Copenhagen, Denmark

Introduction

During the last three decades intrapartum fetal mortality has decreased all over the western world. In most clinics this decrease in fetal mortality rate has been paralleled by an increased frequency of electronic monitoring of the fetus during labor. However, even large randomized trials comparing electronic monitoring. of the fetus to a group of fetuses not being monitored have failed to prove a beneficial effect of electronic monitoring concerning the perinatal mortality. When major neurological handicaps are evaluated in the same series even this condition have not been influenced by electronic monitoring in the few randomized trials ( 1 ). These results may be explained by the few intrapartum fetal deaths and the few neurologically damaged fetuses in both groups. However, it stresses the need for other ways of fetal monitoring.

As the ultimate goal of fetal monitoring is to prevent cell damage of the most vital and most sensitive organs (e.g. brain and heart), a method of fetal monitoring which detects a decrea- 
sed oxygen/energy supply to these organs before cell damage occur is necessary. If the exchange of oxygen and carbon dioxide between the maternal and fetal circulation is diminished (e.g. cord compression, placental insufficiency, hypertone of the uterus) the first change will be a decreased p02 and increased pC02. By definition this is a respiratory acidosis and this condition has been proved to be without deleterious effect to the fetus as long as the oxygen level is sufficient to maintain normal metabolism. However, if the oxygen level decreases further, the metabolism changes from aerobic to anaerobic pass-ways causing accumulation of organic acids and especially lactic acid. In this case the acidosis is changing from a respiratory to a metabolic acidosis (or a combination of the two types of acidosis). Further, in animal experiments it has been shown that metabolic acidosis caused by hypoxia parallels the degree of brain damage (2).

As a consequence of these pathophysiological changes during fetal hypoxia measurement of the fetal acid base state seems to be the best way of assessing fetal well being. Measurement of $\mathrm{pH}$ is a good indicator of acidosis, but it is not possible to evaluate the degree of metabolic/respiratory components if only the $\mathrm{pH}$ is known.

Monitoring of the pC02 might be a good indicator of a beginning acidosis, but if $\mathrm{pH}$ is not known many cases of slight respiratory acidosis - which do not need intervention - might be classified as pathological. Consequently, the ideal would be to monitor both the $\mathrm{pH}$ and the pCO2 simultaneously and continuously.

In the following the results of tissue pH monitoring during labor, transcutaneous pCO2 monitoring during labor, and combined tissue $\mathrm{pH}$ and transcutaneous pCO2 monitoring during labor will be described. 
Tissue $\mathrm{pH}(\mathrm{t} \mathrm{pH})$ monitoring during labor

At present only one electrode has been used for tissue pH monitoring during labor. This electrode (Roche) is a combined glass- and reference electrode which is applied to the fetal head using a special application tool making it possible to apply the electrode without the use of an amnioscope. The electrode is held into position by a spiral electrode, the tip of the glass electrode being situated in the subcutaneous tissue. The $\mathrm{pH}$ is displayed on a pH-meter and on the cardiotocogram as a continuous line or dots every ten seconds. In 229 consecutive tpH monitorings in which the tissue $\mathrm{pH}$ was monitored until delivery the tissue $\mathrm{pH}$ correlated very well to the umbilical artery blood $\mathrm{pH}$ $(r=0.71)$. Tissue $\mathrm{pH}$ values between 7.02 and 7.40 were seen in the serie. All monitorings were performed by the two authors.

The duration of monitoring in all 337 cases was very long in most cases and more than $20 \%$ were monitored 300 - 500 minutes. Most patients were monitored between two and five hours and only 59 patients were monitored for less than 30 minutes. The drift of the electrode did not depend on the duration of monitoring. Sixty-seven per cent of the monitorings were successful, 10\% were partly successful (a stable tracing was obtained but the tissue $\mathrm{pH}$ was not stable until the delivery), and $23 \%$ of the tracings were unsuccessful with no stable tissue pH value obtained. After the first 150 monitorings in which the tissue $p H$ value did not influence the management of labor, the following management of labor was established:

1. In case of severe bradycardia ( $<70$ beats per minute) obstetrical intervention was performed.

2. In all other cases obstetrical intervention was only performed if tissue $\mathrm{pH}$ was $<7.20$ ( 7.15 if delivery could be expected within 15 minutes or if $t-p H$ increased within 15 minutes).

3. A pathological CTG was treated conservatively (e.g. by oxygen, changing of maternal position or by i.v. $\mathrm{NaCl}$ ) 
Seventy-two patients were managed by $t-p H$. In these patients we found a significant reduction of vacuum extraction/outlet forceps deliveries compared to a matched group of patients in which the $\mathrm{pH}$ was not used in the management of labor (25.8 versus $8.6 \%, \mathrm{p}=$ $0.007)$. The state of the neonates did not differ between the two groups ( 3 ).

Transcutaneous pCO2 monitoring during labor

The transcutaneous $\mathrm{pCO2}$ electrode can be applied to the fetal head with a suction or a glue fixation ring. No difference between simultaneously obtained values from a glue fixated electrode and a suction fixated electrode was found (4).

Within the EEC (European Economic Community) a multicenter trial was set up. In this trial all patients were monitored by a Radiometer electrode heated to $41^{\circ} \mathrm{C}$. The study was performed by the authors from Denmark, by Stephan Schmidt and Erich Saling from Berlin, John Spencer and Paul Johnson from Oxford, and Martin Bergmans and Herman van Geijn from Amsterdam. The number of patients were $39,31,50,14$ from the four mentioned centers. The correlation between the umbilical artery blood pCO2 and the transcutaneous pCO2 (tc-pC02) obtained 0-10 minutes before delivery was acceptable, $(n=49, r=0.49)$, but this correlation was not as good at the correlation found by the authors in an earlier study in which an electrode temperature of $44^{\circ} \mathrm{C}$ was used $(r=0.77, n=64)$. The correlation between the arterial umbilical artery. blood pCO2 and transcutaneous pCO2 was best in the centers that were used to tc-pC02 monitoring.

In three acidotic neonates (arterial umbilical artery blood pH less than 7.15) tc-pCO2 was monitored 0 - 10 minutes before delivery. The tc-pCO2 was increased in one case ( $13.3 \mathrm{kPa})$, but it was normal in the other two cases ( 7.5 and $5.1 \mathrm{kPa})$. 
Especially during the last part of labor pCO2 may change rather rapidly. Nevertheless, comparing the tc-pCO2 value obtained until 0-60 minutes before delivery gives an impression of the value of the method as applied by the centers involved. Therefore, we looked at the possibility of predicting neonatal acidosis from the transcutaneous $\mathrm{pCO} 2$ value 0-60 minutes before delivery. The best discrimination was obtained by a pCO2 limit of $8.0 \mathrm{kPa}$, but even then only four out of ten acidatic neonates were found, the six false negatives having tc-pCO2 values evenly distributed between 5 and $8 \mathrm{kPa}$. Further, $31 / 104$ of the neonates with umbilical artery blood $\mathrm{pH}$ above 7.15 had a transcutaneous $\mathrm{pCO} 2$ value above 8.0 .

Consequently, the detection rate was only $40 \%$. An increased tc-pCO2 was found in $30 \%$ of the normal neonates.

If tc-pc02 was normal, the $r$ isk of neonatal acidosis was $6 / 79=$ $8 \%$ - i.e. almost the same as in the total material (risk 10/114 = $9 \%)$. This means that in the EEC-material a low tc-pC02 value 0-60 minutes and even 0-10 minutes before delivery was not reassuring concerning the acid base state of the newborn.

The umbilical artery blood $\mathrm{pCO2}$ and the last transcutaneous pC02 value at the different centers was studied. In most centers the mean umbilical artery blood pC02 was the same (6.6 - $6.7 \mathrm{kPa})$, one center having a mean umbilical artery blood pC02 of 7.1 . In contrast the tc-pCO2 varied between the centers with mean values from 6.8 to $8.0 \mathrm{kPa}$. The highest mean values were found at the two centers with the highest experience in tc-pC02 monitoring. Theoretically, the metabolic production of $\mathrm{CO2}$ of the outer skin layer should contribute to the tc-pCO2 with $0.5 \mathrm{kPa}$ and the tc-pC02 should consequently be $0.5 \mathrm{kPa}$ higher than the umbilical artery pC02. This was nearly the case in the two most experienced departments.

The success rates of tc-pCO2 monitoring was quite different between the centers. In the most experienced center the success rate was close to $100 \%$ in the first state of labor but in one of 
the less experienced centers the success rate was only $75 \%$ in the first stage. In the second stage the highest success rate was $75 \%$ and the lowest only $25 \%$.

The average number of applications during the first stage varied between 1.1 and 1.5 , whereas the average number of applications during the second stage varied much more (0.1 - 1.2 applications). An increased number of applications did not seem to increase the success rate.

All centers had many cases in which the electrode was applied very early in labor, most applications being performed at a cervical dilatation of $3-4 \mathrm{~cm}$.

Monitoring was performed for more than five hours in $10 \%$ of the cases, and only $27 \%$ were monitored for less than one hour.

The only complication seen after the monitoring was a small red spot following suction fixation. No burns caused by the heated electrode was found.

In four cases the umbilical artery blood $\mathrm{pH}$ was less that 7.10 .

In the first case meconium stained liquor was found and Caesarean section was performed because of suspected fetal distress. A child of 2450 gram ( 40 weeks) was delivered. Apgar scores were $6 / 1$ and $10 / 5$. Umbilical artery blood pH was 7.09 but the pc02 was not measured in the umbilical cord blood. The child was intubated and ventilated but did not need transport to the pediatric unit. The tc-pco2 was monitored for four hours but not during the last 30 minutes before delivery. The tc-pco2 value increased from 6.5 $\mathrm{kPa}$ to $13.3 \mathrm{kPa}$ (last value). In this case transcutaneous pco2 predicted fetal acidosis.

In the next case a child was delivered spontaneously with Apgar $8 / 1$, 9/5. The weight was $3290 \mathrm{gram}$ ( 40 weeks). Umbilical artery $\mathrm{pH}$ was 7.01 (vein 7.10), pC02 was $11.6 \mathrm{kPa}$ (vein $8.3 \mathrm{kPa}$ ), and standard base excess was $-9.2 \mathrm{mEq} / 1$ (vein-10.1). Artificial 
ventilation was performed but no intubation was necessary. No transport to pediatric unit. Tc-pco2 monitoring was performed for almost two hours and until birth. The transcutaneous pco2 increased from 8.0 to $13.4 \mathrm{kPa}$ during monitoring. In this case acidosis was predicted by an increasing tc-pco2.

In the third case Caesarean section was performed for suspected fetal distress. Fetal weight was 3000 gram ( 40 weeks). Apgar scores were $7 / 1$, 9/5. Umbilical artery blood pH 7.09 (vein 7.13) pC02 $8.3 \mathrm{kPa}$ (vein 8.2) - SBE-10.4 mEq/1(vein-6.3). No artificial ventilation was needed and the child did not need transport to pediatric unit. Tc-pCo2 monitoring was performed for 95 minutes but was discontinued one hour before birth. The tc-pco2 increased from 5.1 to $9.3 \mathrm{kPa}$. In this case the tc-pco2 increase was only moderate but the monitoring was not performed for the last hour before birth and might had predicted the acidosis more clearly during the last hour before birth.

The last case of severe neonatal acidosis followed Caesarean section performed for dystocia. Fetal weight was 3660 gram (39 weeks), Apgar scores were $6 / 1,9 / 5$. No artificial ventilation was performed and the neonate was not transported to the pediatric unit. $\mathrm{pH}$ was 7.08 (vein 7.14)-pC02 8.0 kPa (vein 7.0) and SBE $-11.7 \mathrm{mEq} / 1(v e i n-10.2)$. Tc-pCO2 monitoring was performed for almost 4 hours, but monitoring was discontinued more than one hour before birth. The tc-pco2 value ( $5.5 \mathrm{kPa})$ did not increase during monitoring. In this case the fetal acidosis could not be predicted by transcutaneous pCo2 monitoring, but monitoring was not performed during the last hour before birth. Further, the case was mostly a case of metabolic acidosis as SBE was low and as pCO2 was almost normal.

Concluding: two out of four cases were. predicted by transcutaneous pCO2 monitoring. The other two cases were not monitored for the last 60 minutes and fetal acidosis might have been predicted if monitoring had been performed throughout labor. 
Continuous base excess monitoring during labor

The technique of continuous base excess monitoring during labor is quite difficult to handle. It is necessary to apply an invasive tissue $\mathrm{pH}$ electrode and at the same time a transcutaneous pCO2 electrode. The tissue $\mathrm{pH}$ value and the $\mathrm{pCO} 2$ value can be noted on the cardiotocogram every ten seconds (e.g. on the labor- and fetal heart rate tracing). At the same time the SBE can be calculated on line using a microcomputer. Following delivery all calculated standard base excess values can be printed out graphically.

In seven cases continuous standard base excess monitoring was performed preceding delivery of a non-acidotic child with normal Apgar scores. In all cases the standard base excess was slightly increasing or constant during delivery (5).

The correlation between the standard base excess of the umbilical artery blood and the calculated standard base excess (calculated from the tissue and the transcutaneous values) was acceptable ( $r=$ $0.66, p<0.05, n=13)(6)$.

In two cases we found continuous standard base excess monitoring of special interest.

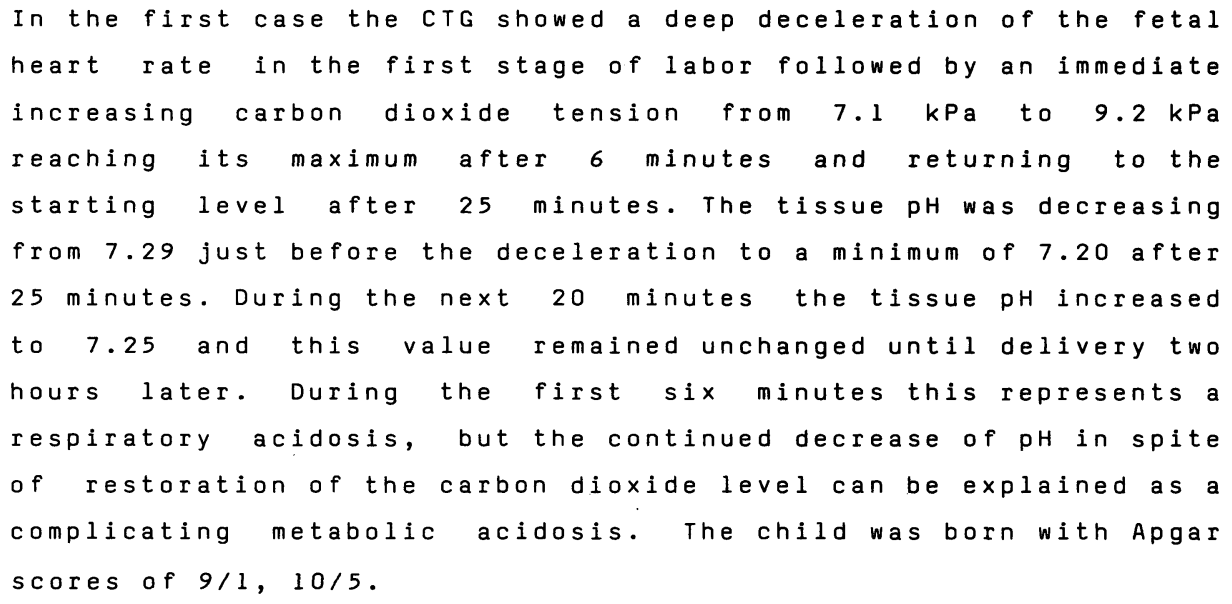




\begin{abstract}
The second example was a patient who had variable decelerations of the fetal heart rate during the second stage of labor. This was accompanied by a gradual tissue pH decrease from a value of 7.28 to below 7.20. The electrode was reapplied and as the tissue value was still below 7.20, vacuum extraction was performed. The carbon dioxide tension was constantly $5.3 \mathrm{kPa}$ during the first 30 minutes but increased to $8.2 \mathrm{kPa}$ during the 1 ast 15 minutes. This example illustrates the development of a metabolic acidosis and the increasing carbon dioxide tension during the last 15 minutes is probably secondary to the metabolic acidosis. Apgar scores were $7 / 1,10 / 3,3 / 5$ (caused by aspiration). Later the infant quickly recovered and no neonatal morbidity was found. Umbilical artery blood $\mathrm{pH}$ was 7.11 and pCO2 was $8.4 \mathrm{kPa}\left(44^{\circ} \mathrm{C}\right.$ value), the standard base excess was $-9.5 \mathrm{mEq} / 1$.
\end{abstract}

Possible future strategies for intrapartum monitoring

At present none of the mentioned ways of monitoring are ready for routine use. If transcutaneous pC02 monitoring becomes easier and future studies show that the sensitivity and specificity of the method becomes better with increased experience (especially decreasing the non-monitored period(s) of the last part of labor), transcutaneous pCO2 monitoring might be applied as a screening method in high risk cases and in cases with pathological CTG. If the tc-pco2 value becomes pathological further evaluation is needed and in this case an invasive method (e.g. a tissue pH electrode) should be applied. Unfortunately, the present tissue $\mathrm{pH}$ electrode can not be used routinely as it is very difficult to handle and is very expensive. If a better tissue pH electrode becomes available (e.g. an improved version of the needle electrode which has shown acceptable performance during animal experiments) (7) the best way of monitoring would consequently be: 
1. CTG and tc-pCO2 monitoring in all high risk cases.

2. In cases with pathological CTG- and/or pathological tc-pCo2monitoring this is supplementet with tissue $\mathrm{pH}$ monitoring with a $\mathrm{pH}$ electrode.

3. Acute intervention for suspected fetal distress is always incated in cases of metabolic acidosis.

4. The obstetrician might try "conservative" treatment (e.g. oxygen, i.v. fluid, change of maternal position) in cases with respiratory acidosis. This should only be tried if spontaneous delivery is expected and actually takes place after a short while - or if the respiratory acidosis is successfully treated by the "conservative" management.

\section{References}

(1) MacDonald D, A Grant, M Sheridan-Pereira, P Boylan, I Chalmers: Am J Obstet Gynecol 152 (1985) 524

(2) Myers RE: Am J Obstet Gynecol 112 (1972) 246

(3) Weber T: Acta Obstet Gynecol Scand 61 (1982) 351

(4) Nickelsen C, T Weber: Brit J Obstet Gynaecol 93 (1986) 1268

(5) Nickelsen C. T Weber: Eur J Obstet Gynecol Reprod Biol 21 (1986) 7

(6) Nickelsen C, SC Thomsen, T Weber: Br J Obstet Gynaecol 92 (1985) 220

(7) Nickelsen C, T Weber, AJ Hansen, A Hansen, T Zeuthen: Int J Gynaecol Obstet 24 (1986) 459 